\title{
Collective Atomic Motion in an Optical Lattice formed inside a High Finesse Cavity
}

\author{
B. Nagorny, Th. Elsässer, and A. Hemmerich \\ Institut für Laser-Physik, Universität Hamburg, Luruper Chaussee 149, D-22761 Hamburg, Germany
}

(Dated: November 21, 2018)

\begin{abstract}
We report on collective non-linear dynamics in an optical lattice formed inside a high finesse ring cavity in a so far unexplored regime, where the light shift per photon times the number of trapped atoms exceeds the cavity resonance linewidth. We observe bistability and self-induced squeezing oscillations resulting from the retro-action of the atoms upon the optical potential wells. We can well understand most of our observations within a simplified model assuming adiabaticity of the atomic motion. Non-adiabatic aspects of the atomic motion are reproduced by solving the complete system of coupled non-linear equations of motion.
\end{abstract}

PACS numbers: 32.80.Pj, 42.50.Vk, 42.62.Fi, 42.50.-p

Single atoms interacting with a few photons inside a high finesse cavity are an extensively studied key system of quantum optics. Only recently the significance of the atomic motion in this model system has been recognized and a wealth of new physics was found including cooling and trapping single atoms by single photons [1, 2]. More recently, it has been pointed out that optical cavities with sufficient finesse and large mode volumes could provide a new means to cool and trap even large atomic samples by coherent scattering, with the promise of extending laser cooling to a wider class of atomic species and possibly even molecules $3,4,4,5,6,6]$. A so far unexplored regime of atom-cavity interactions arises for large atom samples trapped in cavity-enhanced far off-resonant light fields, if the collective coupling strength (i.e. the light shift per photon times the atom number) exceeds the cavity resonance linewidth. For sufficiently high finesse of the cavity, the otherwise tiny retro-action of the moving atoms upon the light field becomes a significant feature of this system. This leads to an inherently collective character of the atomic motion and a corresponding non-linear dynamics of the intra-cavity field. Such collective long-range interactions might allow to create controlled quantum entanglement [8], a perspective possibly useful for quantum computation with neutral atoms. Other intriguing perspectives are the implementation of improved cooling schemes for atomic species otherwise not accessible, for example, as indicated in ref. 7]. Sympathetic cooling without collisions might become possible for low particle densities via thermalization of atom samples trapped at distant locations inside the cavity.

In this paper we explore the collective motion of atoms trapped inside an optical standing wave formed by two mutually counterpropagating travelling wave modes in a high-finesse ring resonator. A fast servo tightly locks the laser frequency in resonance with one of the modes. Within a narrow window around the case of exactly symmetric pumping of both cavity modes, a stable optical lattice is formed. Asymmetric pumping on the order of a few percent yields surprisingly complex dynamics. The most striking feature is a drop of the intra-cavity intensity of the unlocked mode by more than an order of magnitude, if the incoupled powers deviate by only a few percent in favour of the locked mode. This reduced intensity level is maintained up to several ten milliseconds until the slowly decaying lattice population and thus the collective interaction strength fall below a certain value, where the system suddenly jumps back to the mode of operation characteristic for a cavity without atoms. This jump is accompanied by a sudden spatial shift of the lattice. Depending on the total intensity directed to the cavity and the exact degree of pumping asymmetry the bistable behavior can be more or less pronounced and under certain circumstances self-induced radial squeezing oscillations of the atoms arise. Non-linear dynamics due to optical pumping and saturation is known to occur in low-finesse resonators operating close to an atomic resonance 9]. Our lattice operates far from resonance where optical pumping and saturation are negligible and the atoms merely act as a dispersive medium.

Our system is described in detail in ref. [10] and sketched in Fig.1(a). The triangular ring cavity has a finesse of $1.8 \times 10^{5}$, a cavity resonance linewidth of $\gamma_{c} / \pi=17.3 \mathrm{kHz}$ and a large mode volume of $2.6 \mathrm{~mm}^{3}$. The linearly polarized output of a diode laser is servo locked into resonance with one of the travelling wave modes. The laser frequency is red detuned with respect to the D2-line of rubidium $\left({ }^{85} \mathrm{Rb}\right)$ by $0.7 \mathrm{~nm}$. Cold rubidium atoms are provided by a standard magneto-optic trap (MOT). We can observe the powers transmitted through the cavity and perform temperature and position measurements by means of fluorescence imaging. Our intra-cavity optical lattice operates in the regime of strong collective interactions characterized by $U N \geq 1$, where $N$ is the number of trapped atoms and $U \equiv \Delta_{0} / \gamma_{c}$, with $\Delta_{0}=0.091 \mathrm{~s}^{-1}$ being the light shift per photon.

In a typical experimental cycle the MOT superimposed on the lattice loads up to several $10^{6}$ atoms into the lattice, before it is shut off and the atoms are kept by the lattice only. We record the power of the unlocked mode leaking out of the cavity through one of the mirrors as illustrated in Fig.1(a). From the known transmission of the mirror we obtain the intra-cavity intensity $I_{-}$of the unlocked mode. Similarly we can observe the intra-cavity intensity $I_{+}$of the locked mode. We thus measure the scaled quantities $\chi_{ \pm} \equiv I_{ \pm} / I_{0}$, where $I_{0}$ denotes the sum 

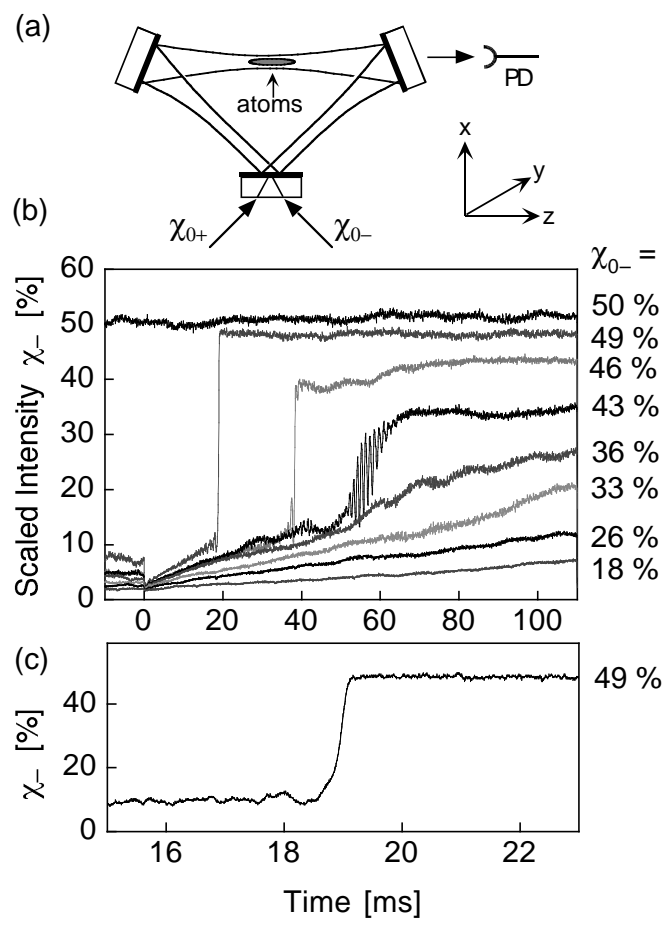

FIG. 1: (a) Sketch of experimental system, PD = photo diode. (b) Transmitted intensity of unlocked mode for $\chi_{0 \pm}=$ $50 \%, 49 \%, 46 \%, 43 \%, 36 \%, 33 \%, 26 \%, 18 \%$. (c) Expanded view of the jump feature in the $49 \%$ trace of (b).

of the intensities in both travelling modes without atoms inside the cavity. We provide adjustable powers $P_{0 \pm}$ to be coupled into the travelling wave modes which we refer to in terms of the scaled quantities $\chi_{0 \pm} \equiv P_{0 \pm} / P_{0}$ with $P_{0}$ being the total power coupled into the cavity. Note that $\chi_{ \pm}=\chi_{0 \pm}$, if no atoms are present inside the cavity. Moreover the fast servo acts to maintain the condition $\chi_{+}=\chi_{0+}$ for any value of the atom-cavity coupling $U N$.

In Fig.1(b) we illustrate the time evolution of the intra-cavity intensity in the unlocked mode. In the uppermost trace we have adjusted perfect symmetric pumping, i.e, $\chi_{0 \pm}=50 \%$. In this case we find a constant value $\chi_{-}=50 \%$ independent of the presence of atoms or the MOT beams which are shut off at $t=0 \mathrm{~ms}$. Both modes build up with equal intensities and a stable optical lattice is formed. This is confirmed by temperature measurements, showing that the atoms are kept for long times at the initial temperature provided by the MOT. If $\chi_{0-}$ is adjusted above $50 \%, \chi_{-}$initially drops to $50 \%$ independent of the exact value of $\chi_{0-}$. As the atom number $N$ decreases in time $\chi_{-}$gradually approaches $\chi_{0-}$. For the locked mode $\chi_{+}=\chi_{0+}$ is maintained at all times. More dramatic consequences arise for values of $\chi_{0-}$ below 50\%. The lower traces in Fig.1(b) are observed for $\chi_{0-}$ adjusted to $49 \%, 46 \%, 43 \%, 36 \%, 33 \%, 26 \%$, and $18 \%$ respectively. The corresponding values of the initial interaction strength $U N(t=0) \approx 4.48,4.25,4.01,3.54$, $3.30,2.95,2.48$ are carefully determined by measuring the initial particle number $N(t=0)$ via fluorescence detec- tion. The observed decrease of $N(t=0)$ with decreasing $\chi_{0}$ - arises because the capture efficiency decreases with the lattice well depth. Note that already during the MOT phase a significantly reduced value of $\chi_{-}$is found. When the MOT is turned off, $\chi_{-}$drops to nearly zero. Subsequently, as the number of trapped atoms decreases due to collisional losses, $\chi_{\text {- gradually increases until a sudden }}$ jump occurs which nearly restores the intensity level $\chi_{0-}$ expected in absence of atoms. The time for this jump to occur increases with decreasing $\chi_{0}$ - while the level to which the intensity initially drops decreases. Moreover, as $\chi_{0-}$ decreases, the jump feature washes out and is not anymore visible below $\chi_{0-}=38 \%$. In the vicinity of the boundary, where the jump feature vanishes, pronounced oscillations in the $\mathrm{kHz}$ range are observed, corresponding to twice the radial vibrational frequency. An example for this behavior is shown in the $43 \%$ trace of Fig.1(b). An expanded view on the jump in the $\chi_{0-}=49 \%$ trace of Fig.1(b) is given in Fig.1(c) showing some residual oscillatory behavior right before the jump. Note that the observed rise time approximately equals a quarter of the radial oscillation time. The observed drop in transmission for the unlocked mode in the traces of Fig.1(b) is accompanied by a corresponding increase of the reflected power, i.e., the overall intra-cavity intensity is reduced in the presence of atoms. Note that in all cases the unperturbed cavity condition $\chi_{+}=\chi_{0+}$ is well kept for the locked mode. We have measured the radial temperature before and after the jump finding $\mathrm{T}=90 \mu \mathrm{K}$ and $\mathrm{T}=$ $142 \mu \mathrm{K}$ respectively. The temperature increase observed is compatible with adiabatic heating expected as a result of the change in potential well depth during the jump. This is plausible since the observed rate of change of the potential well depth satisfies the condition for adiabaticity.

In order to understand the oscillation feature in Fig.1(b) we have measured the atomic position and momentum distributions during this oscillation. The results are plotted in Fig.2(a). We observe an anticyclic variation of the position and momentum spread at twice the radial vibrational frequency. This shows that the origin of the oscillatory feature is a self-induced squeezing oscillation arising for the radial degrees of freedom. This explanation is confirmed by plotting the observed oscillation frequency versus the well depth as shown in Fig.2(b) where the expected square root dependence is found.

We have probed the response of the system to a sudden change of the total power $P_{0}$ coupled to the cavity, which is introduced during a $2 \mathrm{~ms}$ long time window in a region well before the jump occurs. The change of $P_{0}$ is parametrized in terms of the remaining fraction $\xi_{P}$. In Fig.3(a) a typical example of the response is shown for $\xi_{P}=0.5$. As a first immediate reaction to the reduction of $P_{0}$ the intra-cavity intensity in the unlocked mode $I_{-}$ rapidly drops on a time scale given by the cavity decay time $\gamma_{c}{ }^{-1}$, as might be expected. However, subsequently an increased steady state value of $I_{-}$is approached on a much slower time scale. At $t=2 \mathrm{~ms}$ the original value 

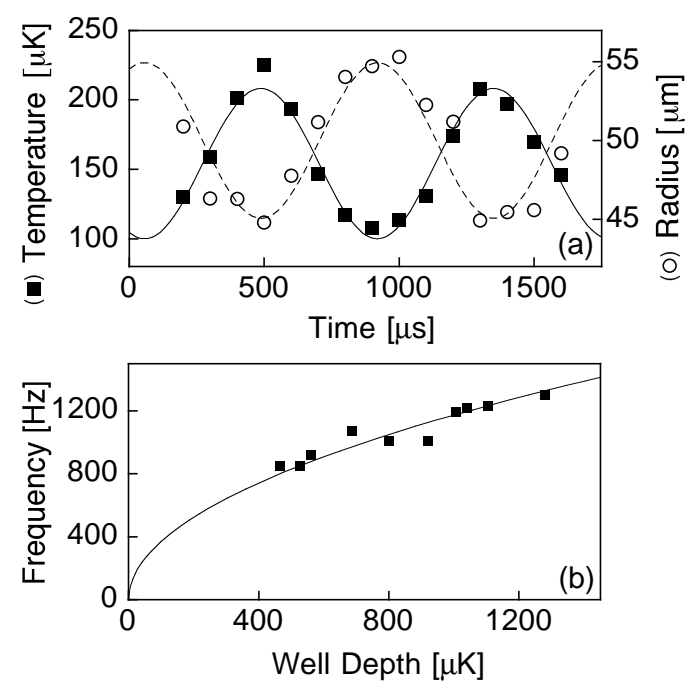

FIG. 2: (a) Oscillation of the atomic position (circles) and momentum spread (rectangles). The solid lines are trigonometric fits with $\pi$ phase delay. (b) Oscillation frequency plotted versus the well depth. The solid line shows the expected square root dependence.
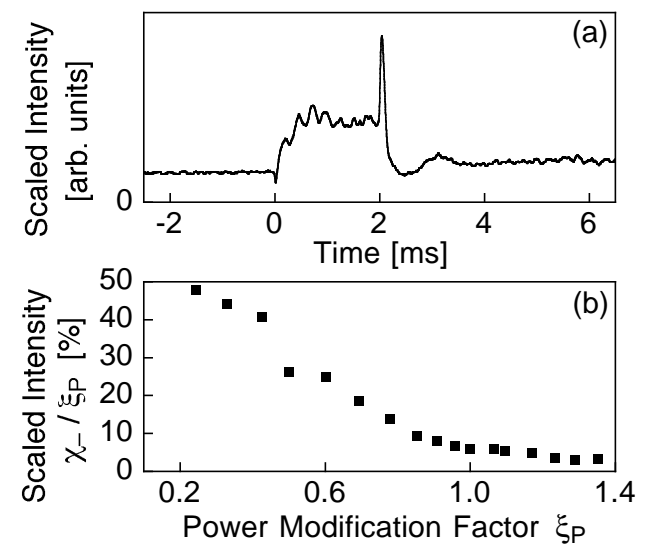

FIG. 3: (a) Intensity $I_{-}$plotted versus time. Between $t=$ $0 \mathrm{~ms}$ and $t=2 \mathrm{~ms}$ the total power coupled to the cavity is reduced by $50 \%$. (b) Plot of the scaled intensity $\chi_{-} / \xi_{P}$ versus the power modification factor $\xi_{P}$.

of $P_{0}$ is reestablished, i.e., $\xi_{P}$ jumps back to 1 . After a fast transient increase, $I_{-}$drops nearly to its original value before the change of $P_{0}$ was introduced. Note also the additional ripple at about $3 \mathrm{kHz}$, which amounts to about six times the radial vibrational frequency. We have recorded similar traces for varying values of $\xi_{P}$. In Fig.3(b) the value of $\chi_{-} / \xi_{P}$ at $t=1.9 \mathrm{~ms}$ is plotted versus $\xi_{P}$, i.e. $I_{-}$is scaled to $I_{0} \xi_{P}$. This graph nicely illustrates the paradox behavior that more external power yields less intra-cavity power.

Our system is classically described by $6 N+2$ coupled non-linear differential equations for the $3 N$ position and momentum coordinates and the two complex electric field amplitudes of the cavity modes [11]. We assume a
Gaussian geometry of the cavity modes with atoms located merely close to the focus. We can combine the two equations for the fields into a single equation because the servo lock keeps the field amplitude of the locked mode at a constant value. A significant part of the experimental findings can be explained by a simple model based on the assumption of a thermal sample of atoms adiabatically adjusting to the potential well depths and positions. We may thus eliminate $6 \mathrm{~N}$ equations of motion concerned with the external degrees of freedom and remain with a single non-linear differential equation for the intra-cavity complex electric field amplitude $a(t)$ of the unlocked mode scaled to $\sqrt{I_{0}}$. Leaving the details of its derivation to a forthcoming publication we merely indicate the result here:

$$
\begin{array}{r}
\frac{d}{d \tau} a=i \frac{U N}{\sqrt{\chi_{0+}}} L(a)|a| a-a+ \\
+\sqrt{\chi_{0-}}-i U N \sqrt{\chi_{0+}} L(a) \frac{a}{|a|}, \\
L(a) \equiv e^{-\eta_{a x} \sqrt{\frac{\left|a_{0}\right|}{|a|}} \frac{1}{1+\eta_{\text {rad }} \frac{\sqrt{\chi_{0+}}+\left|a_{0}\right|}{\sqrt{\chi_{0+}}+|a|}}}
\end{array}
$$

In this equation $\tau \equiv \gamma_{c} t$ and $a_{0} \equiv a(t=0)$. The function $L(a)$ is comprised of two factors describing the axial and radial degrees of localization. The truncation parameters $\eta_{a x}$ and $\eta_{\text {rad }}$ are defined as the ratios between the thermal energy $k_{B} T$ and the well depth at time $t=0$ for the axial and radial directions respectively. Eq. (1) can be readily integrated numerically, using the initial values $a_{0}$ prepared by the MOT according to the traces of Fig.1(b). In the time dependence of $N$ we account for linear losses due to collisions with hot background atoms and losses due to inelastic two-body collisions with rates measured in our experiment. The values of $\eta_{a x}$ and $\eta_{\text {rad }}$ are determined by temperature measurements to be approximately 0.5 and 0.3 respectively with about 0.1 uncertainty. The difference is due to different well depths in axial and radial directions at $\mathrm{t}=0$.

In Fig.4(a) and (b) we show simulations excellently matching our experimental findings in Fig.1(b) and (c). For the initial atom-cavity coupling strength $U N(t=0)$ we used the values $2.38,2.23,2.15,1.75$, which fall within a few percent of those determined for the corresponding experimental traces, however reduced by a common scaling factor 1.89. The need for this factor is not surprising, because in our atom number measurements up to a factor two uncertainty should be expected for the absolute values, while relative values are on the few percent level. The basic characteristics of the jump feature, i.e. the time for the jump to occur and the rise time, are well reproduced. Eq. (1) lets us also calculate the phase $\phi$ of the complex field $a(t)$ showing that the jump is accompanied by a rapid shift of the optical lattice of approximately a fraction $\lambda / 10$, where $\lambda$ is the wavelength of the lattice light. This is illustrated by the dashed trace in Fig.4(b). 

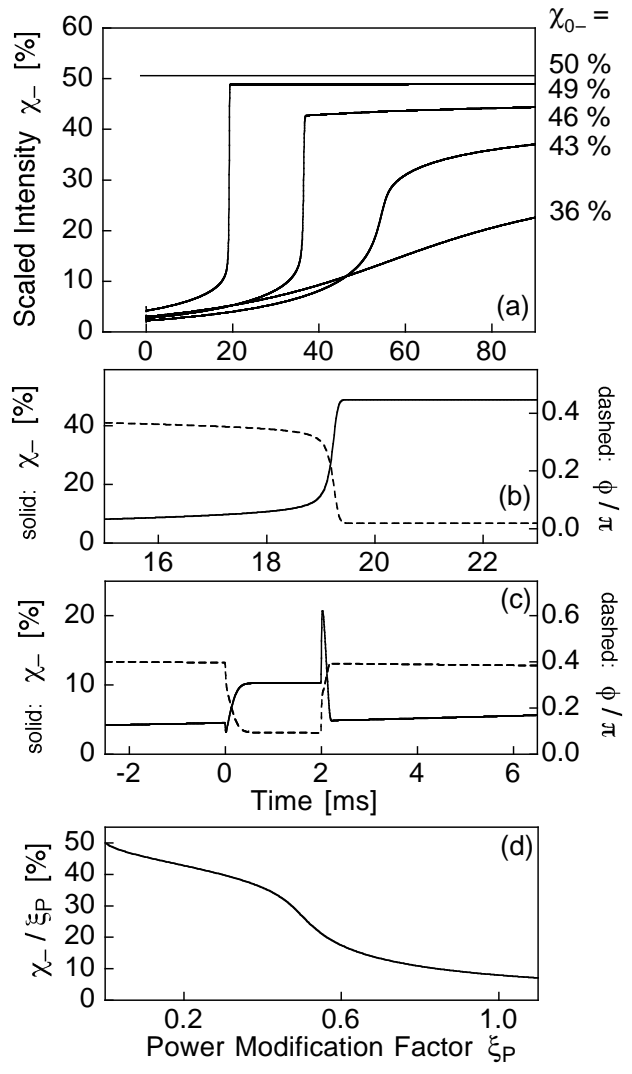

FIG. 4: Theoretical simulations assuming adiabatic atomic motion. The graphs in (a), (b), (c) and (d) correspond to the experimental observations in Fig.1(b) Fig.1(c), Fig.3(a), and Fig.3(b).

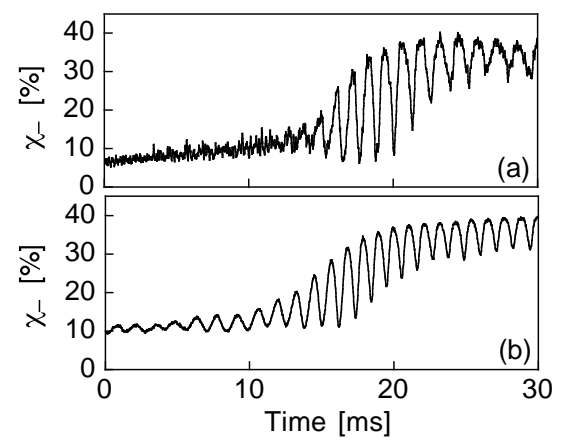

FIG. 5: (a) Experimental observation of squeezing oscillation. (b) Simulation of the squeezing oscillations of (a) by means of solving the general equations of motion for hundred atoms.
As shown in Fig.4(c) and (d) we may also reproduce the findings of Fig.3. Again we calculated the corresponding phase of $a(t)$, finding that also the change of $P_{0}$ yields a spatial shift of the lattice.

We may not expect that our simple model can account for non-adiabatic motion as for example the squeezing oscillations shown in Fig.1(b) and Fig.5(a). Non-adiabatic motion is particularly expected to occur with regard to the radial degrees of freedom, which are not very tightly bound. In order to admit non-adiabatic dynamics we have solved the complete system of coupled equations of motion for hundred particles including axial and radial degrees of freedom. The result shown in Fig.5(b) nicely reproduces the frequency observed in (a). The calculation even reproduces the fact that the frequency of the squeezing oscillation slightly decreases with time, a detail that we cannot explain so far. Apart from such squeezing oscillations the results obtained by the full calculations are in good agreement with those of the adiabatic model.

In summary, we have studied collective atomic motion and corresponding non-linear phenomena arising in an optical lattice formed inside a high finesse ring cavity. The spatial phase of the lattice is not pinned by the phases of the incoupled laser beams, but rather determined by the collective atom-cavity interaction. The bistable behavior found experimentally for asymmetric pumping can be understood in terms of an adiabatic approximation for the atomic motion, whereas the general equations of motion must be considered to model selfinduced squeezing oscillations arising in a certain parameter range. In this article we have only discussed selected aspects of the system dynamics. A wealth of further interesting phenomena could be studied, as for example collective atomic recoil lasing (CARL) 12] which is expected to occur for unidirectional pumping of the cavity.

\section{Acknowledgments}

This work has been supported by Deutsche Forschungsgemeinschaft (DFG) under contract number $H e 2334 / 3-2$. We are grateful to Helmut Ritsch for discussions and numerical support.
[1] P.W.H. Pinkse, T. Fischer, P. Maunz, and G. Rempe, Nature 404, 365-368 (2000).

[2] C. Hood et al., Science 287, 1457 (2000).

[3] A. C. Doherty, A. S. Parkins, S. M. Tan, and D. F. Walls, Phys. Rev. A. 56, 833 (1997).

[4] P. Horak et al., Phys. Rev. Lett. 79, 4974 (1997).

[5] G. Hechenblaikner, M. Gangl, P. Horak, and H. Ritsch,
Phys. Rev. A. 58, 3030 (1998).

[6] H.W. Chan, A.T. Black, and V. Vuletic, Phys. Rev. Lett. 90, 063003 (2003).

[7] Th. Elsässer, B. Nagorny, and A. Hemmerich, Phys. Rev. A 67, 051401(R) (2003).

[8] A. Hemmerich, Phys. Rev. A. 60, 943 (1999).

[9] A. Lambrecht, E. Giacobino, and J.M. Courty, Opt. 
Commun. 115, 199 (1995).

[10] B. Nagorny et al., Phys. Rev. A 67, 031401(R) (2003).

[11] M. Gangl and H. Ritsch, Phys. Rev. A. 61, 043405 (2000).

[12] R. Bonifacio, L. De Salvo, L. M. Narducci, and E. J.
D'Angelo, Phys. Rev. A 50, 1716 (1994), D. Kruse, C. von Cube, C. Zimmermann, and Ph.W. Courteille, quant-ph/0305033 (2003). 